University of Windsor

Scholarship at UWindsor

Physics Publications

Department of Physics

2000

\title{
Lithium isotope shifts as a measure of nuclear size
}

Z. C. Yan

Gordon W. F. Drake

University of Windsor

Follow this and additional works at: https://scholar.uwindsor.ca/physicspub

Part of the Physics Commons

\section{Recommended Citation}

Yan, Z. C. and Drake, Gordon W. F.. (2000). Lithium isotope shifts as a measure of nuclear size. Physical Review A - Atomic, Molecular, and Optical Physics, 61 (2), 225041-225048.

https://scholar.uwindsor.ca/physicspub/130

This Article is brought to you for free and open access by the Department of Physics at Scholarship at UWindsor. It has been accepted for inclusion in Physics Publications by an authorized administrator of Scholarship at UWindsor. For more information, please contact scholarship@uwindsor.ca. 


\title{
Lithium isotope shifts as a measure of nuclear size
}

\author{
Zong-Chao Yan $^{1}$ and G. W. F. Drake ${ }^{2}$ \\ ${ }^{1}$ Steacie Institute for Molecular Sciences, National Research Council of Canada, Ottawa, Ontario, Canada K1A OR6 \\ ${ }^{2}$ Department of Physics, University of Windsor, Windsor, Ontario, Canada N9B 3P4
}

(Received 5 August 1999; published 11 January 2000)

\begin{abstract}
The isotope shifts for $2{ }^{2} P_{J}-2{ }^{2} S$ and $3{ }^{2} S-2{ }^{2} S$ transition energies in lithium are calculated variationally in Hylleraas coordinates, including nonrelativistic, relativistic, and QED terms up to $O(\mu / M), O(\mu / M)^{2}$, $O\left(\alpha^{2} \mu / M\right)$, and $O\left(\alpha^{3} \mu / M\right)$ atomic units, and the lowest-order finite nuclear size correction. With highprecision isotope shift measurements, our results can potentially yield a precise determination of the nuclear charge radius for different isotopes of lithium, and especially for the exotic ${ }^{11} \mathrm{Li}$ " halo" isotope. For the case of ${ }^{7} \mathrm{Li}-{ }^{6} \mathrm{Li}$, using the nuclear charge radii from nuclear scattering data, our calculated isotope shifts for the $2{ }^{2} P_{1 / 2}-2{ }^{2} S, 2{ }^{2} P_{3 / 2}-2{ }^{2} S$, and $3{ }^{2} S-2{ }^{2} S$ transitions are $10534.31(61)(6) \mathrm{MHz}, 10534.70(61)(6) \mathrm{MHz}$, and $11454.31(39)(5) \mathrm{MHz}$, respectively, where the first brackets indicate the uncertainties due to the nuclear charge radii, and the second brackets indicate the computational uncertainties. The experimental isotope shifts are inconsistent with each other and with theory for these transitions.
\end{abstract}

PACS number(s): 31.30.Gs, 31.30.Jv, 21.10.Ft

\section{INTRODUCTION}

The root-mean-square (rms) radius of the nuclear charge distribution is a quantity of fundamental importance in nuclear physics [1]. For lithium, although the rms nuclear radii of the stable species ${ }^{6} \mathrm{Li}$ and ${ }^{7} \mathrm{Li}$ are determined at the $1 \%$ level [2], for unstable ${ }^{8} \mathrm{Li},{ }^{9} \mathrm{Li}$, and ${ }^{11} \mathrm{Li}$ the rms charge radii are unknown. Among these isotopes, the study of ${ }^{11} \mathrm{Li}$ is of great current interest $[3,4]$ because this isotope consists of a ${ }^{9} \mathrm{Li}$ core with a "halo" of two loosely bound neutrons orbiting the nucleus. In addition to the traditional nuclear scattering method, the nuclear charge distribution can be probed by a combination of atomic physics theory and experiment, provided both theory and measurement can be carried out to sufficiently high accuracy. One advantage of such an approach is its nuclear model independence. Although the influence of a finite nuclear charge distribution on the energy level of an atom is well known (see Ref. [5] for a review), it was pointed out by Drake [6] that a high-precision measurement of an isotope shift for a chosen transition might be used to extract the rms nuclear radius. Although the QED terms for light atoms are comparable in size to the nuclear size corrections, they are, to a first approximation, independent of the nuclear mass, and so they largely cancel from the calculated isotope shift. The significance of the method is therefore that the nuclear radius can be determined independently of QED uncertainties. This method was recently applied successfully to the studies of ${ }^{4} \mathrm{He}-{ }^{3} \mathrm{He}[1,7]$ and ${ }^{7} \mathrm{Li}^{+}-{ }^{6} \mathrm{Li}^{+}$[8] isotope shifts. The determined rms radii for ${ }^{3} \mathrm{He}$ and ${ }^{6} \mathrm{Li}^{+}$ are in good agreement with nuclear scattering data, but with substantially improved accuracy.

For ${ }^{6} \mathrm{Li}$ and ${ }^{7} \mathrm{Li}$, Vadla et al. [9] measured the isotope shifts of several transition lines using resonant Doppler-free two-photon laser spectroscopy. For the $3{ }^{2} S-2{ }^{2} S$ transition, the accuracy is $0.17 \%$. Using laser-atomic-beam spectroscopy, Windholz and Umfer [10] measured the isotope shifts for the $2{ }^{2} P_{J}-2{ }^{2} S$ transitions. The precisions they obtained are $28 \mathrm{ppm}$ for the $D_{1}$ line $(J=1 / 2)$ and $114 \mathrm{ppm}$ for the $D_{2}$ line $(J=3 / 2)$. Later, they improved their experiment [11] and reduced the uncertainties to $14 \mathrm{ppm}$ for both lines. The third recent measurements for the isotope shifts in the $2{ }^{2} P_{J}-2{ }^{2} S$ transitions were done by Sansonetti et al. [12] using Doppler-free frequency-modulation spectroscopy. The precision they achieved is $30 \mathrm{ppm}$ for the $D_{1}$ line and 21 ppm for the $D_{2}$ line (reduced to one standard deviation). Although for the $D_{1}$ line the value of Sansonetti et al. and the Windholz et al. revised value agree with each other, there is a discrepancy of $160 \mathrm{ppm}$ for the $D_{2}$ line.

One purpose of this paper is to report the theoretical results of the ${ }^{7} \mathrm{Li}-{ }^{6} \mathrm{Li}$ isotope shifts for the $3^{2} S-2{ }^{2} S$ and $2{ }^{2} P_{J}-2{ }^{2} S$ transitions. A second purpose is to provide a formula for the isotope shift between any two $\mathrm{Li}$ isotopes as a function of the assumed rms nuclear radii. This is motivated by the experimental efforts currently under way at GSI, Darmstadt [4], to measure the Li $3{ }^{2} S-2{ }^{2} S$ isotope shift with a proposed accuracy of $200 \mathrm{kHz}$. The ultimate goal is to determine the rms radii of $\mathrm{Li}$ isotopes, particularly the halo nucleus ${ }^{11} \mathrm{Li}$, with a precision of $10 \%$ or better. The calculations are based on our recent advances [13-19] in highprecision variational calculations for lithium and lithiumlike ions using multiple basis sets in Hylleraas coordinates.

The paper is organized as follows. In Sec. II we review the theoretical formulation of the problem and construction of basis sets in Hylleraas coordinates for the lithium atom wave function. Finite nuclear mass effects and the mass polarization operator are taken into account up to second order by perturbation theory. Especially important are relativistic recoil terms of order $\alpha^{2} \mu / M$ a.u., since it is the accuracy of these terms that limits the accuracy of the final results. Section III presents results for basis sets containing up to 3502 terms, together with a general formula for the determination of rms nuclear radii for any isotope of lithium relative to a chosen reference isotope. Section IV discusses the comparison with experiment for the ${ }^{7} \mathrm{Li}-{ }^{6} \mathrm{Li}$ isotope shifts, and Sec. $\mathrm{V}$ presents some concluding remarks.

\section{THEORETICAL FORMULATION}

After rescaling distances according to $r \rightarrow(m / \mu) r$, the Hamiltonian for a three-electron atomic system is 


$$
H=H_{0}+\lambda H^{\prime},
$$

with

$$
H_{0}=-\frac{1}{2} \sum_{i=1}^{3} \nabla_{i}^{2}-Z \sum_{i=1}^{3} \frac{1}{r_{i}}+\sum_{i>j}^{3} \frac{1}{r_{i j}}
$$

and

$$
H^{\prime}=\sum_{i>j}^{3} \nabla_{i} \cdot \nabla_{j}
$$

in units of $2 R_{M}$, where $R_{M}=(1-\mu / M) R_{\infty}, \mu=m M /(m$ $+M)$ is the electron reduced mass, and $\lambda=-\mu / M$, which can be treated as a perturbation parameter. The Schrödinger equation

$$
H \Psi=E \Psi
$$

can be solved perturbatively by expanding $\Psi$ and $E$ according to

$$
\begin{gathered}
\Psi=\Psi_{0}+\lambda \Psi_{1}+\cdots, \\
E=\varepsilon_{0}+\lambda \varepsilon_{1}+\lambda^{2} \varepsilon_{2}+\cdots .
\end{gathered}
$$

Thus Eq. (4) becomes

$$
\begin{gathered}
H_{0} \Psi_{0}=\varepsilon_{0} \Psi_{0}, \\
\left(\varepsilon_{0}-H_{0}\right) \Psi_{1}=\left(H^{\prime}-\varepsilon_{1}\right) \Psi_{0} .
\end{gathered}
$$

$\varepsilon_{1}$ and $\varepsilon_{2}$ are

$$
\begin{gathered}
\varepsilon_{1}=\left\langle\Psi_{0}\left|H^{\prime}\right| \Psi_{0}\right\rangle, \\
\varepsilon_{2}=\left\langle\Psi_{0}\left|H^{\prime}\right| \Psi_{1}\right\rangle-\varepsilon_{1}\left\langle\Psi_{0} \mid \Psi_{1}\right\rangle .
\end{gathered}
$$

Both $\Psi_{0}$ and $\Psi_{1}$ were solved variationally in multiple basis sets in Hylleraas coordinates containing terms of the form

$$
\begin{aligned}
r_{1}^{j_{1}} & r_{2}^{j_{2}} r_{3}^{j_{3}} r_{12}^{j_{12}} r_{23}^{j_{23}} r_{31}^{j_{31}} e^{-\alpha r_{1}-\beta r_{2}-\gamma r_{3}} \\
& \times \mathcal{Y}_{\left(l_{1} l_{2}\right) l_{12}, l_{3}}^{L M}\left(\hat{\mathbf{r}}_{1}, \hat{\mathbf{r}}_{2}, \hat{\mathbf{r}}_{3}\right) \chi_{1},
\end{aligned}
$$

where $\mathcal{Y}_{\left(l_{1} l_{2}\right) l_{12}, l_{3}}^{L M}$ is a vector-coupled product of spherical harmonics for the three electrons to form a state of total angular momentum $L$, and $\chi_{1}$ is a spin function with spin angular momentum $1 / 2$. As described previously [13,18], all terms from Eq. (11) are nominally included such that

$$
j_{1}+j_{2}+j_{3}+j_{12}+j_{23}+j_{31} \leqslant \Omega,
$$

and the convergence of the eigenvalues is studied as $\Omega$ is progressively increased. Further details may be found in Ref. [18]. Since Eq. (6) is expressed in units of $(1+\lambda) 2 R_{\infty}$, the explicit mass dependence of $E$ is

$$
\begin{aligned}
E= & \varepsilon_{0}+\lambda\left(\varepsilon_{0}+\varepsilon_{1}\right)+\lambda^{2}\left(\varepsilon_{1}+\varepsilon_{2}\right) \\
& +O\left(\lambda^{3}\right) \text { in units of } 2 R_{\infty} .
\end{aligned}
$$

The lowest-order relativistic corrections of $O\left(\alpha^{2}\right)$ and the spin-dependent anomalous magnetic moment corrections of $O\left(\alpha^{3}\right)$ can be written in the form $[20,21]$ (in atomic units)

$$
\Delta E_{\text {rel }}=\left\langle\Psi\left|H_{\text {rel }}\right| \Psi\right\rangle_{J},
$$

where $\Psi$ is a nonrelativistic wave function, and $H_{\text {rel }}$ is defined by

$$
\begin{aligned}
H_{\mathrm{rel}}= & B_{1}+B_{2}+B_{3 e}+B_{3 z}+B_{5}-\pi \alpha^{2} \\
& \times \sum_{i>j}^{3}\left(1+\frac{8}{3} \mathbf{s}_{i} \cdot \mathbf{s}_{j}\right) \delta\left(\mathbf{r}_{i j}\right)+\frac{1}{2} Z \pi \alpha^{2} \sum_{i=1}^{3} \delta\left(\mathbf{r}_{i}\right) \\
& +\frac{m}{M}\left(\widetilde{\Delta}_{2}+\widetilde{\Delta}_{3 z}\right)+\gamma\left(2 B_{3 z}+\frac{4}{3} B_{3 e}+\frac{2}{3} B_{3 e}^{(1)}+2 B_{5}\right) \\
& +\gamma \frac{m}{M} \widetilde{\Delta}_{3 z} .
\end{aligned}
$$

In Eq. (15),

$$
\begin{gathered}
B_{1}=-\frac{\alpha^{2}}{8}\left(\nabla_{1}^{4}+\nabla_{2}^{4}+\nabla_{3}^{4}\right), \\
B_{2}=\frac{\alpha^{2}}{2} \sum_{i>j}^{3}\left[\frac{1}{r_{i j}} \nabla_{i} \cdot \nabla_{j}+\frac{1}{r_{i j}^{3}} \mathbf{r}_{i j} \cdot\left(\mathbf{r}_{i j} \cdot \nabla_{i}\right) \nabla_{j}\right], \\
B_{3 e}=\frac{\alpha^{2}}{2} \sum_{i \neq j}^{3} \frac{1}{r_{i j}^{3}} \mathbf{r}_{j i} \times \mathbf{p}_{i} \cdot\left(\mathbf{s}_{i}+2 \mathbf{s}_{j}\right),
\end{gathered}
$$

$$
\begin{gathered}
B_{3 z}=\frac{Z \alpha^{2}}{2} \sum_{i=1}^{3} \frac{1}{r_{i}^{3}} \mathbf{r}_{i} \times \mathbf{p}_{i} \cdot \mathbf{s}_{i}, \\
B_{5}=\alpha^{2} \sum_{i>j}^{3}\left[\frac{1}{r_{i j}^{3}}\left(\mathbf{s}_{i} \cdot \mathbf{s}_{j}\right)-\frac{3}{r_{i j}^{5}}\left(\mathbf{r}_{i j} \cdot \mathbf{s}_{i}\right)\left(\mathbf{r}_{i j} \cdot \mathbf{s}_{j}\right)\right], \\
\widetilde{\Delta}_{2}=\frac{i Z \alpha^{2}}{2} \sum_{j=1}^{3}\left[\frac{1}{r_{j}} \mathbf{p} \cdot \nabla_{j}+\frac{1}{r_{j}^{3}} \mathbf{r}_{j} \cdot\left(\mathbf{r}_{j} \cdot \mathbf{p}\right) \boldsymbol{\nabla}_{j}\right], \\
\widetilde{\Delta}_{3 z}=Z \alpha^{2} \sum_{i=1}^{3} \frac{1}{r_{i}^{3}} \mathbf{r}_{i} \times \mathbf{p} \cdot \mathbf{s}_{i}, \\
B_{3 e}^{(1)}=\frac{\alpha^{2}}{2} \sum_{i \neq j}^{3} \frac{1}{r_{i j}^{3}} \mathbf{r}_{j i} \times \mathbf{p}_{i} \cdot\left(\mathbf{s}_{i}-\mathbf{s}_{j}\right),
\end{gathered}
$$

with $\mathbf{p}=\mathbf{p}_{1}+\mathbf{p}_{2}+\mathbf{p}_{3}$, and $\gamma$ is

$$
\gamma=\frac{\alpha}{2 \pi}+(-0.32847)\left(\frac{\alpha}{\pi}\right)^{2}+\cdots .
$$

For doublet states, the operator $-\pi \alpha^{2} \Sigma_{i>j}\left(1+\frac{8}{3} \mathbf{s}_{i}\right.$ $\left.\cdot \mathbf{s}_{j}\right) \delta\left(\mathbf{r}_{i j}\right)$ can be replaced by $\pi \alpha^{2} \Sigma_{i>j} \delta\left(\mathbf{r}_{i j}\right)$, and the expectation value of the spin-spin term $B_{5}$ vanishes. $B_{i}$ are the Breit-Pauli terms, the terms proportional to $m / M$ are the 
nuclear relativistic recoil corrections, and the terms proportional to $\gamma$ are the anomalous magnetic moment corrections.

The perturbing effect of mass polarization on the expectation values of Breit operators can be obtained using

$$
\Psi=\Psi_{0}+\lambda\left(\Psi_{1}-\left\langle\Psi_{1} \mid \Psi_{0}\right\rangle \Psi_{0}\right)+\cdots
$$

where the extra term $-\left\langle\Psi_{1} \mid \Psi_{0}\right\rangle \Psi_{0}$ is added to $\Psi_{1}$ so that the first two terms of the right-hand side are orthogonal to each other [22]. Thus, for a Breit operator $A$, one has

$$
\langle\Psi|A| \Psi\rangle=a_{0}+\lambda a_{1}+\cdots,
$$

where

$$
a_{0}=\left\langle\Psi_{0}|A| \Psi_{0}\right\rangle
$$

and

$$
a_{1}=2\left\langle\Psi_{0}|A| \Psi_{1}\right\rangle-2\left\langle\Psi_{0} \mid \Psi_{1}\right\rangle\left\langle\Psi_{0}|A| \Psi_{0}\right\rangle
$$

Furthermore, due to the use of $\mu$-scaled atomic units in Eq. (1), the units of $\langle\Psi|A| \Psi\rangle$ in Eq. (26) are $(\mu / m)^{n} 2 R_{\infty}$, where $-n$ is the degree of homogeneity of operator $A$ in three-electron coordinate space such that

$$
A\left(\beta \mathbf{r}_{1}, \beta \mathbf{r}_{2}, \beta \mathbf{r}_{3}\right)=\beta^{-n} A\left(\mathbf{r}_{1}, \mathbf{r}_{2}, \mathbf{r}_{3}\right) .
$$

Using

$$
\left(\frac{\mu}{m}\right)^{n}=(1+\lambda)^{n} \approx 1+n \lambda
$$

one has the explicit mass-dependent formula

$$
\langle\Psi|A| \Psi\rangle=a_{0}+\lambda\left(n a_{0}+a_{1}\right)+O\left(\lambda^{2}\right) \quad \text { in units of } 2 R_{\infty} .
$$

The QED corrections can be calculated according to the formulation of McKenzie and Drake [23]:

$$
\Delta E_{\mathrm{QED}}=\Delta E_{L, 1}+\Delta E_{L, 2} \text {. }
$$

In Eq. (32), $\Delta E_{L, 1}$ is given by

$$
\Delta E_{L, 1}=\alpha^{3} Z\left[\frac{x F\left(1 s_{1 / 2}\right)+F\left(n l_{j}\right) / n^{3}}{x+\delta_{l, 0} / n^{3}}\right]\left\langle\delta\left(\mathbf{r}_{i}\right)\right\rangle_{1 s^{x} n l},
$$

where $x$ is the number of $1 s$ electrons, $F\left(n l_{j}\right)$ is the oneelectron QED function defined by Johnson and Soff [24], and $\left\langle\delta\left(\mathbf{r}_{i}\right)\right\rangle$ denotes $\left\langle\sum_{i=1}^{3} \delta\left(\mathbf{r}_{i}\right)\right\rangle$. The two-electron QED shift is

$$
\Delta E_{L, 2}=\alpha^{3}\left(\frac{14}{3} \ln \alpha+\frac{164}{15}\right)\left\langle\delta\left(\mathbf{r}_{i j}\right)\right\rangle-\frac{14}{3} \alpha^{3} Q,
$$

where the $Q$ term is defined by

$$
Q=(1 / 4 \pi) \lim _{\epsilon \rightarrow 0}\left\langle r_{i j}^{-3}(\epsilon)+4 \pi(\gamma+\ln \epsilon) \delta\left(\mathbf{r}_{i j}\right)\right\rangle .
$$

In Eq. (35), $\gamma$ is Euler's constant, $\epsilon$ is the radius of a sphere about $r_{i j}=0$ excluded from the integration, and a summation over $i>j$ from 1 to 3 is assumed. Finally, the last correction to be included is that due to finite nuclear size. It is given in lowest order by

$$
\Delta E_{\mathrm{nuc}}=\frac{2 \pi Z r_{\mathrm{rms}}^{2}}{3}\left\langle\delta\left(\mathbf{r}_{i}\right)\right\rangle
$$

where $r_{\mathrm{rms}}=R_{\mathrm{rms}} / a_{\mathrm{Bohr}}, R_{\mathrm{rms}}$ is the root-mean-square radius of the nuclear charge distribution, and $a_{\mathrm{Bohr}}$ is the Bohr radius. A mass scaling factor of $(\mu / m)^{3}$ is included in the definition of $\left\langle\delta\left(\mathbf{r}_{i}\right)\right\rangle$.

In a nonrelativistic approximation, the $\lambda \epsilon_{0}$ term of Eq. [13] is called the normal isotope shift, and the remaining terms $\lambda \epsilon_{1}+\cdots$ are sometimes called the specific isotope shift. However, this partition becomes somewhat artificial when relativistic corrections are included because the overall multiplying factor is no longer simply $1+\lambda$ from the finitemass Rydberg. The $B_{1}$ term scales with mass as $(1+\lambda)^{4}$ and the other terms in Eq. [15] scale as $(1+\lambda)^{3}$.

\section{CALCULATIONS AND RESULTS}

Table I shows a convergence study of $\varepsilon_{0}$ for $3{ }^{2} S$ as the size of basis set increases progressively up to 3502 terms, together with comparisons with King's result [25] using Hylleraas coordinates, and the result of Wang et al. [26] using a full-core-plus-correlation wave function. Our calculation improves theirs by factors of $1.2 \times 10^{6}$ and $2.3 \times 10^{4}$ respectively. A similar convergence study for the $2^{2} S$ and $2{ }^{2} P$ states can be found in Ref. [18]. Our results for the nonrelativistic energies for the $2{ }^{2} S, 2{ }^{2} P$, and $3{ }^{2} S$ are calculated to a computational accuracy of a few parts in $10^{12}$. Table II contains the nonrelativistic energy $\lambda$-expansion coefficients $\varepsilon_{0}, \varepsilon_{1}$, and $\varepsilon_{2}$ for these states.

The expectation values of the Breit operators and the twoelectron $Q$ term were evaluated for the $2{ }^{2} S, 2{ }^{2} P_{J}$, and $3{ }^{2} S$ states of lithium, together with the first-order finite nuclear mass corrections according to Eq. (31). The results are presented in Table III, and a comparison is made with the work of Wang et al. [26] for the $3{ }^{2} S$ state. For the $2{ }^{2} S$ and $2{ }^{2} P_{J}$ states, a comparison with Chung's work [27] can be found in Ref. [19].

Table IV lists the contributions to the $3{ }^{2} S-2{ }^{2} S$ and $2^{2} P_{J}-2{ }^{2} S$ transition energies, from the nonrelativistic, the lowest-order relativistic, and the lowest-order QED terms up to $\mu / M,(\mu / M)^{2},(\mu / M) \alpha^{2}$, and $(\mu / M) \alpha^{3}$, as well as the contributions from the finite nuclear size. The leading isotope-independent term is not included because it does not contribute to the isotope shift for the transition energies. Combining all the coefficients of $\mu / M$, we arrive at the following formulas giving the Li isotope shifts for the $3{ }^{2} S$ $-2{ }^{2} S, 2{ }^{2} P_{1 / 2}-2{ }^{2} S$, and $2{ }^{2} P_{3 / 2}-2{ }^{2} S$ transitions: 
TABLE I. Convergence of the nonrelativistic energy for the $1 s^{2} 3 s^{2} S$ state of lithium, in atomic units. $R(\Omega)$ is the ratio between two successive differences.

\begin{tabular}{rrrrr}
\hline \hline$\Omega$ & No. of terms & \multicolumn{1}{c}{$\varepsilon_{0}(\Omega)$} & $\varepsilon_{0}(\Omega)-\varepsilon_{0}(\Omega-1)$ & $R(\Omega)$ \\
\hline 3 & 51 & -7.353807225055 & & \\
4 & 121 & -7.354068196845 & -0.000260971790 & \\
5 & 257 & -7.354095840826 & -0.000027643980 & 9.440 \\
6 & 503 & -7.354098120924 & -0.000002280098 & 12.124 \\
7 & 919 & -7.354098382175 & -0.000000261251 & 8.727 \\
8 & 1590 & -7.354098417067 & -0.000000034892 & 7.487 \\
9 & 2626 & -7.354098420627 & -0.000000003559 & 9.803 \\
10 & 3502 & -7.354098421082 & -0.000000000455 & 7.821 \\
$\infty$ & \multicolumn{7}{c}{$-7.354098421149(18)$} & & \\
\end{tabular}

${ }^{\mathrm{a}}$ King, Ref. [25].

${ }^{\mathrm{b}}$ Wang et al., Ref. [26].

$$
\begin{aligned}
f_{3{ }^{2} S-2{ }^{2} S}= & -0.13376715(64)(\mu / M) \\
& +0.12364810(29)(\mu / M)^{2} \\
& -0.6666463(55) r_{\mathrm{rms}}^{2}+1.9802(19) r_{\mathrm{rms}}^{2}(\mu / M),
\end{aligned}
$$

$$
\begin{aligned}
f_{2{ }^{2} P_{1 / 2}-2{ }^{2} S}= & -0.12299378(79)(\mu / M) \\
& -0.00395(14)(\mu / M)^{2} \\
& -1.04561095(89) r_{\mathrm{rms}}^{2} \\
& +2.136(15) r_{\mathrm{rms}}^{2}(\mu / M), \\
f_{2{ }^{2} P_{3 / 2}-2{ }^{2} S=} & -0.12299837(79)(\mu / M) \\
& -0.00395(14)(\mu / M)^{2} \\
& -1.04561095(89) r_{\mathrm{rms}}^{2} \\
& +2.136(15) r_{\mathrm{rms}}^{2}(\mu / M),
\end{aligned}
$$

in units of $2 R_{\infty}$. Table $\mathrm{V}$ summarizes the nuclear data for the various isotopes of lithium, including the values of $\mu / M$ (in terms of the atomic mass $M_{A}$ ) and $R_{\text {rms }}$ for the isotopes ${ }^{7} \mathrm{Li}$ and ${ }^{6} \mathrm{Li}$. With these values as input, Table VI lists the calculated contributions to the isotope shifts for the $2{ }^{2} P_{J}-2^{2} S$ transitions. The first uncertainties are due to the uncertainties of the rms nuclear radii, and the second uncertainties are from the uncertainties of the computed coefficients. The main uncertainties of the total isotope shifts are due to the nuclear charge radii, since the terms of order $\mu / M,(\mu / M)^{2}$, and $\alpha^{2} \mu / M$ are well established, and the QED terms of order $\alpha^{3} \mu / M$ hardly affect the comparison. The contribution from the $r_{\text {rms }}^{2} \mu / M$ term is negligible.

Table VII contains the contributions to the isotope shift for the $3{ }^{2} S-2{ }^{2} S$ transition. King [25] also studied this isotope shift using Hylleraas type wave functions. His result is $-0.381800 \mathrm{~cm}^{-1}$. However, his value for the expectation value of the mass polarization operator for the $3{ }^{2} S$ state is only accurate to about $270 \mathrm{ppm}$, and he did not include the relativistic recoil term of order $\alpha^{2} \mu / M$. The accuracy of this latter term is in fact the dominant source of theoretical uncertainty.

\section{Determination of nuclear radii}

The principal motivation for this work is to provide a spectroscopic means of determining nuclear radii from the observed isotope shifts. For this purpose, we take the calculated coefficients in Eqs. (37) to (39) as correct and rewrite these equations in the form

$$
R_{\mathrm{rms}}^{2}\left({ }^{A} \mathrm{Li}\right)=R_{\mathrm{rms}}^{2}\left({ }^{6} \mathrm{Li}\right)+\frac{E_{\text {meas }}^{A}-E_{0}^{A}}{C},
$$

for the nuclear radius squared of an arbitrary isotope ${ }^{A} \mathrm{Li}$ relative to ${ }^{6} \mathrm{Li}$. $E_{\text {meas }}^{A}$ is the measured isotope shift for ${ }^{A} \mathrm{Li}$ relative to ${ }^{6} \mathrm{Li}$, and $E_{0}^{A}$ contains all the calculated contribu-

TABLE II. Nonrelativistic eigenvalue coefficients $\varepsilon_{0}, \varepsilon_{1}$, and $\varepsilon_{2}$ for the $1 s^{2} 2 s^{2} S, 1 s^{2} 3 s^{2} S$, and $1 s^{2} 2 p^{2} P$ states of lithium.

\begin{tabular}{clll}
\hline \hline Coefficient & \multicolumn{1}{c}{$1 s^{2} 2 s^{2} S$} & \multicolumn{1}{c}{$1 s^{2} 3 s^{2} S$} & \multicolumn{1}{c}{$1 s^{2} 2 p^{2} P$} \\
\hline$\varepsilon_{0}$ & $-7.4780603236503(71)$ & $-7.354098421149(18)$ & $-7.410156531763(42)$ \\
$\varepsilon_{1}$ & $-0.30184278302(25)$ & $-0.29203983410(22)$ & $-0.2467388875(70)$ \\
$\varepsilon_{2}$ & $-1.49978867(17)$ & $-1.38594352(23)$ & $-1.55884(14)$ \\
\hline \hline
\end{tabular}


TABLE III. $\lambda=-\mu / M$ expansion coefficients $a_{0}$ and $n a_{0}+a_{1}$ in Eq. (31) of the expectation values of the Breit operators and the two-electron $Q$ term for the $1 s^{2} 2 s^{2} S, 1 s^{2} 3 s^{2} S$, and $1 s^{2} 2 p^{2} P_{1 / 2}$ states of lithium. Units are $2 R_{\infty}$.

\begin{tabular}{|c|c|c|}
\hline Operator & $\lambda^{0}$ & $\lambda^{1}$ \\
\hline \multicolumn{3}{|c|}{$1 s^{2} 2 s^{2} S$} \\
\hline$B_{1}$ & $-0.004183221020(30)$ & $-0.01700684(50)$ \\
\hline$B_{2}$ & $-0.0000231961868(73)$ & $-0.0002334493(20)$ \\
\hline$\tilde{\Delta}_{2}$ & $-0.00697140748(15)$ & $-0.0231834107(30)$ \\
\hline$\left\langle\delta\left(\mathbf{r}_{i}\right)\right\rangle$ & $13.842609642(55)$ & $42.012420(30)$ \\
\hline$\left\langle\delta\left(\mathbf{r}_{i j}\right)\right\rangle$ & $0.54432979(31)$ & $1.5509735(93)$ \\
\hline \multicolumn{3}{|c|}{$1 s^{2} 3 s^{2} S$} \\
\hline$B_{1}$ & $-0.0041460166(18)$ & $-0.01685232(40)$ \\
\hline$B_{2}$ & $\begin{array}{l}-0.000022893198(20) \\
-0.0000230^{\mathrm{a}}\end{array}$ & $-0.000224300(30)$ \\
\hline$\tilde{\Delta}_{2}$ & $-0.006892527026(74)$ & $-0.022833349(50)$ \\
\hline$\left\langle\delta\left(\mathbf{r}_{i}\right)\right\rangle$ & $13.73650959(87)$ & $41.69727(30)$ \\
\hline$\left\langle\delta\left(\mathbf{r}_{i j}\right)\right\rangle$ & $\begin{array}{l}0.53617515(47) \\
0.564^{\mathrm{a}}\end{array}$ & $1.530145(40)$ \\
\hline$Q$ & $0.015794(96)$ & $-0.0844(60)$ \\
\hline$B_{1}+Z \pi \alpha^{2}\left\langle\delta\left(\mathbf{r}_{i}\right)\right\rangle / 2$ & $\begin{array}{c}-0.0006989613(18) \\
-0.0006968^{\mathrm{a}} \\
1 s^{2} 2 p^{2} P_{1 / 2}\end{array}$ & \\
\hline$B_{1}$ & $-0.0041272804330(40)$ & $-0.016819160(65)$ \\
\hline$B_{2}$ & $-0.0000211102385(48)$ & $-0.00022313(10)$ \\
\hline$B_{3 e}$ & $0.00000401499220(99)$ & $-0.000001208(50)$ \\
\hline$B_{3 z}$ & $-0.00000503010104(65)$ & $-0.000000053(30)$ \\
\hline$B_{3 e}^{(1)}$ & $0.0000024783902(69)$ & $0.0000026243(30)$ \\
\hline$\tilde{\Delta}_{2}$ & $-0.006848916194(77)$ & $-0.0228543(20)$ \\
\hline$\tilde{\Delta}_{3 z}$ & $0.000001799199(82)$ & $-0.000006162(50)$ \\
\hline$\left\langle\delta\left(\mathbf{r}_{i}\right)\right\rangle$ & $13.67619549(13)$ & $41.6725(24)$ \\
\hline$\left\langle\delta\left(\mathbf{r}_{i j}\right)\right\rangle$ & $0.53228142(51)$ & $1.53011(10)$ \\
\hline$Q$ & $0.0229975(88)$ & $-0.0795(20)$ \\
\hline
\end{tabular}

${ }^{a}$ Wang et al., Ref. [26].

TABLE IV. Expansion coefficients of $\lambda, \lambda^{2}, r_{\text {rms }}^{2}$, and $\lambda r_{\text {rms }}^{2}$ for the lithium $1 s^{2} 3 s^{2} S-1 s^{2} 2 s^{2} S$ and $1 s^{2} 2 p^{2} P_{J}-1 s^{2} 2 s^{2} S$ transition energies, where $\lambda=-\mu / M$. The subscript indicates the value of $J$. Units are $2 R_{\infty}$.

\begin{tabular}{llll}
\hline \hline Term & \multicolumn{1}{c}{ Source } & \multicolumn{1}{c}{$3{ }^{2} S-2{ }^{2} S$} & \multicolumn{1}{c}{$2{ }^{2} P_{J}-2{ }^{2} S$} \\
\hline$\lambda$ & Nonrelativistic & $0.13376485142(33)$ & $0.1230076874(70)$ \\
$\lambda^{2}$ & Nonrelativistic & $0.12364810(29)$ & $-0.00395(14)$ \\
$\lambda$ & Relativistic, $\alpha^{2}$ & $0.00000222(64)$ & $-0.00001634(79)_{1 / 2}$ \\
& & & $-0.00001175(79)_{3 / 2}$ \\
$\lambda$ & Anomalous magnetic, $\alpha^{3}$ & & $-0.00000000205(10)_{1 / 2}$ \\
& & & $0.00000000102(10)_{3 / 2}$ \\
$\lambda$ & One-electron QED, $\alpha^{3}$ & -0.000000046 & 0.000002312 \\
$\lambda$ & Two-electron QED, $\alpha^{3}$ & $0.000000132(14)$ & $0.0000001232(98)$ \\
$r_{\text {rms }}^{2}$ & Finite nuclear size & $-0.6666463(55)$ & $-1.04561095(89)$ \\
$\lambda r_{\text {rms }}^{2}$ & Finite nuclear size & $-1.9802(19)$ & $-2.136(15)$ \\
\hline \hline
\end{tabular}


TABLE V. Summary of the nuclear spin $(S)$, lifetime $\left(T_{1 / 2}\right)$, atomic mass $\left(M_{A}\right)$, magnetic dipole and electric quadrupole nuclear moments $\left(\mu_{I}\right.$ and $Q$ ), hyperfine structure splitting (HFS, in the $2 S$ state), rms mass radius $R_{\mathrm{rms}}^{(m)}$, and charge radius $R_{\mathrm{rms}}^{(e)}$ for the isotopes of lithium.

\begin{tabular}{lccccc}
\hline \hline Quantity & ${ }^{6} \mathrm{Li}$ & ${ }^{7} \mathrm{Li}$ & ${ }^{8} \mathrm{Li}$ & ${ }^{9} \mathrm{Li}$ & ${ }^{11} \mathrm{Li}$ \\
\hline$S$ & 1 & $3 / 2$ & 2 & $3 / 2$ & $3 / 2$ \\
$T_{1 / 2}(\mathrm{~ms})^{\mathrm{a}}$ & $\infty$ & $\infty$ & $838(6)$ & $178.3(4)$ & $8.59(14)$ \\
$M_{A}(\mathrm{u})^{\mathrm{b}}$ & $6.0151223(5)$ & $7.0160040(5)$ & $8.0224867(5)$ & $9.0267891(21)$ & $11.043796(29)$ \\
$\mu_{I}(\mathrm{~nm})^{\mathrm{c}}$ & $0.8220473(6)$ & $3.2564268(17)$ & $1.653560(18)$ & $3.4391(6)$ & $3.6678(25)$ \\
$Q(\mathrm{mbarn})^{\mathrm{d}}$ & $-0.83(8)$ & $-40.0(3)$ & $31.1(5)$ & $-27.4(1.0)$ & $-31.2(4.5)$ \\
$\mathrm{HFS}(\mathrm{MHz})^{\mathrm{e}}$ & $228.205259(3)$ & $803.5040866(10)$ & $382.543(7)$ & $856(16)$ & $920(39)$ \\
$R_{\mathrm{rms}}^{(m)}(\mathrm{fm})^{\mathrm{f}}$ & $2.35(3)$ & $2.35(3)$ & $2.38(2)$ & $2.32(2)$ & $3.10(17)$ \\
$R_{\mathrm{rms}}^{(e)}(\mathrm{fm})^{\mathrm{g}}$ & $2.55(4)$ & $2.39(3)$ & $?$ & $?$ & $?$ \\
\hline \hline
\end{tabular}

${ }^{\text {a Reference [28]. }}$

${ }^{\mathrm{b}}$ Reference [29]. For Li, $\mu / M=1 /\left[1822.888511(43) M_{A}-2\right]$.

${ }^{\mathrm{c}}$ Reference [30].

${ }^{\mathrm{d}}$ See Ref. [31] for ${ }^{6} \mathrm{Li}$, Ref. [32] for ${ }^{7} \mathrm{Li}$, and Ref. [33] for ${ }^{8} \mathrm{Li},{ }^{9} \mathrm{Li}$, and ${ }^{11} \mathrm{Li}$.

${ }^{\text {e}}$ See Ref. [34] for ${ }^{6} \mathrm{Li}$ and ${ }^{7} \mathrm{Li}$, Ref. [35] for ${ }^{8} \mathrm{Li}$, and Ref. [36] for ${ }^{9} \mathrm{Li}$ and ${ }^{11} \mathrm{Li}$.

${ }^{\mathrm{f}}$ Reference [37].

${ }^{\mathrm{g}}$ Reference [2].

tions to the isotope shift except for the nuclear size contributions. The constant $C$ is given by

$$
\begin{aligned}
C & =\frac{2 \pi Z}{3}\left[\left\langle\delta\left(\mathbf{r}_{i}\right)\right\rangle_{\mathrm{i}}-\left\langle\delta\left(\mathbf{r}_{i}\right)\right\rangle_{\mathrm{f}}\right] \\
& =-2.4565 \mathrm{MHz} / \mathrm{fm}^{2} \text { for } 2{ }^{2} P_{J}-2{ }^{2} S \\
& =-1.5661 \mathrm{MHz} / \mathrm{fm}^{2} \text { for } 3{ }^{2} S-2{ }^{2} S .
\end{aligned}
$$

It depends on the transition $i \rightarrow f$ in question, but it is nearly independent of the mass number $A$. Using the atomic masses from Table V, the numerical values for $E_{0}^{A}$ are listed in Table VIII. In the case of ${ }^{11} \mathrm{Li}$, the uncertainty in $E_{0}^{A}$ for the $3{ }^{2} S-$ $2{ }^{2} S$ transition is composed of a $\pm 0.18 \mathrm{MHz}$ uncertainty from the theoretical coefficients (mainly the $\alpha^{2} \mu / M$ term), and a $\pm 0.12 \mathrm{MHz}$ uncertainty from the ${ }^{11} \mathrm{Li}$ atomic mass. The final uncertainty of $\pm 0.21 \mathrm{MHz}$ is sufficient to determine $R_{\mathrm{rms}}^{2}$ for ${ }^{11} \mathrm{Li}$ to an accuracy of $\pm 0.13 \mathrm{fm}^{2}$. Somewhat higher accuracy in $R_{\mathrm{rms}}^{2}$ could be obtained from the $2{ }^{2} P_{J}$ $-2^{2} S$ transitions $\left( \pm 0.10 \mathrm{fm}^{2}\right)$, but the larger linewidth would pose additional experimental difficulties. With further improvements to the theory, a better measurement of $M_{A}$ for ${ }^{11} \mathrm{Li}$ would also be desirable in order to exploit fully the isotope shift method of measuring the nuclear charge radius.

\section{COMPARISONS WITH EXPERIMENT}

Our result for the $3{ }^{2} S-2{ }^{2} S{ }^{7} \mathrm{Li}-{ }^{6} \mathrm{Li}$ isotope shift of $11454.29(38)(5) \mathrm{MHz}$ lies just at the upper edge of the error limits for the value $11434(20) \mathrm{MHz}$ measured by Vadla

TABLE VI. Contributions to the ${ }^{7} \mathrm{Li}-{ }^{6} \mathrm{Li}$ isotope shifts for the $1 s^{2} 2 p^{2} P_{J}-1 s^{2} 2 s^{2} S$ transitions and comparison with experiment. Units are MHz.

\begin{tabular}{lcc}
\hline \hline Contribution & $2{ }^{2} P_{1 / 2}-2{ }^{2} S$ & $2{ }^{2} P_{3 / 2}-2{ }^{2} S$ \\
\hline$\mu / M$ & Theory & \\
$(\mu / M)^{2}$ & $10533.50181(60)^{\mathrm{a}}$ & $10533.50181(60)^{\mathrm{a}}$ \\
$\alpha^{2} \mu / M$ & $0.0573(20)$ & $0.0573(20)$ \\
$\alpha^{3} \mu / M$, anom. magnetic & $-1.397(66)$ & $-1.004(66)$ \\
$\alpha^{3} \mu / M$, one-electron & $-0.0001753(84)$ & $0.0000875(84)$ \\
$\alpha^{3} \mu / M$, two-electron & 0.198 & 0.198 \\
$r_{\text {rms }}^{2}$ & $0.01055(84)$ & $0.01055(84)$ \\
$r_{\text {rms }}^{2} \mu / M$ & $1.94(61)$ & $-0.00073(11)$ \\
Total & $-0.00073(11)$ & $10534.70(61)(7)$ \\
Sansonetti et al. $[12]$ & $10534.31(61)(7)$ & $10533.3(2)$ \\
Windholz and Umfen [10] & Experiment & $10539.9(1.2)$ \\
Scherf et al. [11] & $10532.9(3)$ & $10534.93(15)$ \\
\hline \hline
\end{tabular}

The additional uncertainty from the atomic mass determinations is $\pm 0.008 \mathrm{MHz}$. 
TABLE VII. Contributions to the ${ }^{7} \mathrm{Li}-{ }^{6} \mathrm{Li}$ isotope shift for the $1 s^{2} 3 s^{2} S-1 s^{2} 2 s^{2} S$ transition. Units are MHz.

\begin{tabular}{lc}
\hline \hline Contribution & $3{ }^{2} S-2^{2} S$ \\
\hline$\mu / M$ & $11454.668686(29){ }^{a}$ \\
$(\mu / M)^{2}$ & $-1.7938640(41)$ \\
$\alpha^{2} \mu / M$ & $0.188(53)$ \\
$\alpha^{3} \mu / M$, one electron & -0.00395 \\
$\alpha^{3} \mu / M$, two electron & $0.0113(12)$ \\
$r_{\text {rms }}^{2}$ & $1.24(39)$ \\
$r_{\text {rms }}^{2} \mu / M$ & $-0.000677(98)$ \\
Total & $11454.31(39)(5)$ \\
King [25] & 11446.1 \\
Vadla et al. (experiment) [9] & $11434(20)$ \\
\hline \hline
\end{tabular}

${ }^{\mathrm{a}}$ The additional uncertainty from the atomic mass determinations is $\pm 0.008 \mathrm{MHz}$.

et al. [9] (see Table VII). Here, the experimental precision of $\pm 20 \mathrm{MHz}$ is not sufficient to provide a significant test of theory beyond the lowest order $\mu / M$ term, and the uncertainty is much larger than the nuclear radius contribution.

However, as illustrated in Fig. 1, the interpretation of the measurements for the $2{ }^{2} P_{J}-2{ }^{2} S$ isotope shifts is much more obscure for several reasons. First, as a preliminary remark, we have verified in parallel calculations for the $1 s 2 p^{3} P_{J}-1 s 2 s^{3} S_{1}$ isotope shift in $\mathrm{Li}^{+}$that theory and experiment are in good agreement. In fact, the $\mathrm{Li}^{+}$experiment determines the difference in nuclear radii to be $R_{\text {rms }}\left({ }^{6} \mathrm{Li}\right)$ $-R_{\text {rms }}\left({ }^{7} \mathrm{Li}\right)=0.15 \pm 0.01 \mathrm{fm}$, in close agreement with the value $0.16 \pm 0.05 \mathrm{fm}$ from nuclear scattering data. We therefore take the values for $R_{\text {rms }}$ listed in Table V as correct. For the case of neutral lithium, the two sets of measurements by Sansonetti et al. [12] and Scherf et al. [11] do not agree with each other, and of the four separate measurements, only the $2^{2} P_{3 / 2}-2{ }^{2} S$ result of Scherf et al. is in reasonable agreement with theory. In Fig. 1, note that the large outer error bars on the two theoretical data points are due to the nuclear radius uncertainty. These error bars are directly correlated since a change in $R_{\mathrm{rms}}^{2}$ would shift both theoretical points in the same direction and by the same amount, as further discussed in the following paragraph.

A useful check on the experimental data is provided by the $2{ }^{2} P_{3 / 2}-2{ }^{2} P_{1 / 2}$ splitting isotope shift (SIS), obtained from the difference between the $2^{2} P_{3 / 2}-2{ }^{2} S$ and $2{ }^{2} P_{1 / 2}-$ $2{ }^{2} S$ isotope shifts. To the necessary accuracy, this quantity

TABLE VIII. Values of $E_{0}^{A}$ to determine $R_{\mathrm{rms}}^{2}$ from the measured isotope shift in various transitions (see Eq. [40]). Units are MHz.

\begin{tabular}{cccc}
\hline \hline Isotopes & $E_{0}^{A}\left(2{ }^{2} P_{1 / 2}-2{ }^{2} S\right)$ & $E_{0}^{A}\left(2{ }^{2} P_{3 / 2}-2{ }^{2} S\right)$ & $E_{0}^{A}\left(3{ }^{2} S-2{ }^{2} S\right)$ \\
\hline${ }^{7} \mathrm{Li}-{ }^{6} \mathrm{Li}$ & $10532.37(7)$ & $10532.76(7)$ & $11453.07(6)$ \\
${ }^{8} \mathrm{Li}-{ }^{6} \mathrm{Li}$ & $18473.18(12)$ & $18473.87(12)$ & $20088.23(10)$ \\
${ }^{9} \mathrm{Li}-{ }^{6} \mathrm{Li}$ & $24631.53(16)$ & $24632.44(16)$ & $26785.18(13)$ \\
${ }^{10} \mathrm{Li}-{ }^{6} \mathrm{Li}$ & $29575.97(20)$ & $29577.07(20)$ & $32162.12(17)$ \\
${ }^{11} \mathrm{Li}-{ }^{6} \mathrm{Li}$ & $33615.77(24)$ & $33617.02(24)$ & $36555.34(21)$ \\
\hline \hline
\end{tabular}

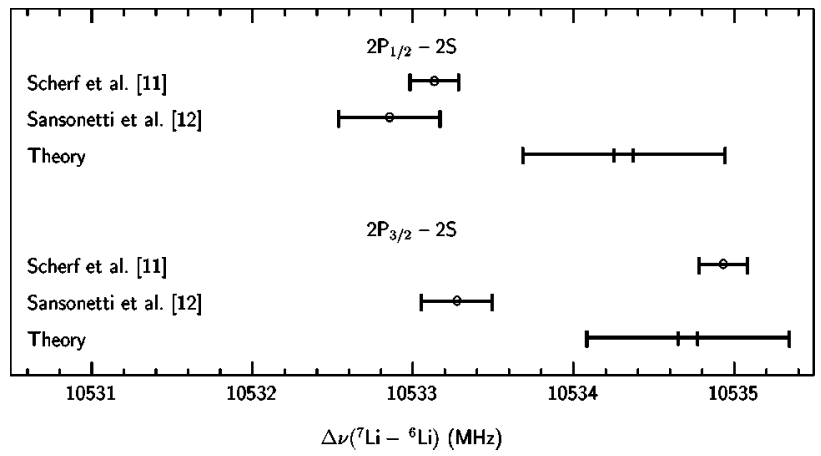

FIG. 1. Comparison between theory and experiment for the ${ }^{7} \mathrm{Li}-$ ${ }^{6} \mathrm{Li} 2{ }^{2} P_{J}-2{ }^{2} S$ isotope shift. For the theoretical data points, the inner error bars denote the computational uncertainty, and the outer error bars include the nuclear radius uncertainty. The outer error bars for the two theoretical data points are directly correlated (see the text). Experimental error bars represent one standard deviation.

is determined entirely by the spin-dependent part of the single $\alpha^{2} \mu / M$ term in Table VI. QED and finite nuclear volume corrections largely cancel since they are nearly the same for both states. The predicted SIS is $0.393(66) \mathrm{MHz}$, in excellent agreement with the measured value 0.4(3) $\mathrm{MHz}$ from Sansonetti et al. It may be that their SIS is more accurate than their error estimates for the full transition frequencies would indicate, but that both measurements are too low by about 1.4(3) MHz. On the other hand, the data of Scherf et al. yield a SIS of $1.80(15) \mathrm{MHz}$, indicating that at least one of their measurements is incorrect by about nine standard deviations. It is clear that additional experimental work is required to resolve these discrepancies.

\section{DISCUSSION}

In this work, we have obtained the theoretical data necessary to derive nuclear radii from isotope shift measurements in neutral lithium. This work complements earlier work for transitions in $\mathrm{Li}^{+}$[8] where theory and experiment were shown to be in good agreement, and consistent with the nuclear radii for ${ }^{6} \mathrm{Li}$ and ${ }^{7} \mathrm{Li}$ derived from nuclear scattering measurements. The results can be applied directly to the ${ }^{11} \mathrm{Li}$ "halo" isotope for which the rms nuclear charge radius is difficult to determine by standard methods because of its low abundance. The present theoretical accuracy would allow a measurement of $R_{\mathrm{rms}}^{2}$ accurate to $\pm 0.13 \mathrm{fm}^{2}$. Further improvements to the theory would also require an improved atomic mass measurement for ${ }^{11} \mathrm{Li}$ in order to exploit to the full the potential accuracy of the isotope shift method.

The comparison between theory and experiment for the ${ }^{7} \mathrm{Li}-{ }^{6} \mathrm{Li}$ isotope shift in the $2{ }^{2} P_{J}-2{ }^{2} S$ transitions is not very satisfactory. The experiments are inconsistent with each other and with theory. A resolution of these inconsistencies would be very valuable in verifying that all contributions to isotope shifts in lithium have been calculated correctly and to sufficient accuracy.

Note added in proof. In recent calculations for helium, Pachucki and Sapirstein [38] discuss additional QED recoil corrections of order $\alpha^{3} \mu / M$ which are not included in the present work. Similar corrections should also be included for 
lithium, but they are unlikely to change the present results for the isotope shifts by more than a few tenths of a megahertz.

\section{ACKNOWLEDGMENTS}

We are grateful to Andreas Dax (GSI) for encouraging this project and communicating his proposal for the ${ }^{11} \mathrm{Li}$ halo nucleus measurement. This work was supported by the Natu- ral Sciences and Engineering Research Council of Canada. Z.C.Y. was also supported by the National Science Foundation through a grant for the Institute for Theoretical Atomic and Molecular Physics at Harvard University and the Smithsonian Astrophysical Observatory.
[1] D. Shiner, R. Dixson, and V. Vedantham, Phys. Rev. Lett. 74, 3553 (1995).

[2] C. W. de Jager, H. de Vries, and C. de Vries, At. Data Nucl. Data Tables 14, 479 (1974).

[3] I. Tanihata, J. Phys. G 22, 157 (1996).

[4] A. Dax (private communication). See also http://wwwaix.gsi.de/laserweb/lithium/jahres.html.

[5] J. D. Morgan III and J. S. Cohen, in Atomic, Molecular, and Optical Physics Handbook, edited by G. W. F. Drake (AIP, New York, 1996).

[6] G. W. F. Drake, in Long-Range Casimir Forces: Theory and Recent Experiments on Atomic Systems, edited by F. S. Levin and D. A. Micha (Plenum, New York, 1993).

[7] F. Marin, F. Minardi, F. Pavone, M. Inguscio, and G. W. F. Drake, Z. Phys. D: At., Mol. Clusters 32, 285 (1995).

[8] E. Riis, A. G. Sinclair, O. Poulsen, G. W. F. Drake, W. R. C. Rowley, and A. P. Levick, Phys. Rev. A 49, 207 (1994).

[9] C. Vadla, A. Obrebski, and K. Niemax, Opt. Commun. 63, 288 (1987).

[10] L. Windholz and C. Umfer, Z. Phys. D: At., Mol. Clusters 29, 121 (1994).

[11] W. Scherf, O. Khait, H. Jäger, and L. Windholz, Z. Phys. D: At., Mol. Clusters 36, 31 (1996).

[12] C. J. Sansonetti, B. Richou, R. Engleman, Jr., and L. J. Radziemski, Phys. Rev. A 52, 2682 (1995).

[13] Z.-C. Yan and G. W. F. Drake, Phys. Rev. A 52, 3711 (1995).

[14] G. W. F. Drake and Z.-C. Yan, Phys. Rev. A 52, 3681 (1995).

[15] Z.-C. Yan and G. W. F. Drake, Phys. Rev. A 52, R4316 (1995).

[16] Z.-C. Yan and G. W. F. Drake, J. Phys. B 30, 4723 (1997).

[17] Z.-C. Yan and G. W. F. Drake, Phys. Rev. Lett. 79, 1646 (1997).

[18] Z.-C. Yan, M. Tambasco, and G. W. F. Drake, Phys. Rev. A 57, 1652 (1998).
[19] Z.-C. Yan and G. W. F. Drake, Phys. Rev. Lett. 81, 774 (1998).

[20] A. P. Stone, Proc. Phys. Soc. London 77, 786 (1961); 81, 868 (1963).

[21] G. W. F. Drake and Z.-C. Yan, Phys. Rev. A 46, 2378 (1992).

[22] L. I. Schiff, Quantum Mechanics, 3rd ed., (McGraw-Hill, New York, 1968).

[23] D. K. McKenzie and G. W. F. Drake, Phys. Rev. A 44, R6973 (1991); 48, 4803(E) (1993).

[24] W. R. Johnson and G. Soff, At. Data Nucl. Data Tables 33, 405 (1985).

[25] F. W. King, Phys. Rev. A 40, 1735 (1989); 43, 3285 (1991).

[26] Z.-W. Wang, X.-W. Zhu, and K. T. Chung, Phys. Rev. A 46, 6914 (1992).

[27] K. T. Chung, Phys. Rev. A 44, 5421 (1991); Z.-W. Wang, X.-W. Zhu, and K. T. Chung, Phys. Scr. 47, 65 (1993).

[28] G. Audi, O. Persillon, J. Blachot, and A. H. Wapstra, Nucl. Phys. A 624, 1 (1997).

[29] G. Audi and A. H. Wapstra, Nucl. Phys. A 595, 409 (1995).

[30] LBNL Isotopes Project—LUNDS Universitet Nuclear Data Dissemination Home Page http://ie.lbl.gov/toi.html.

[31] D. Sundholm, P. Pyykkö, L. Laaksonen, and A. J. Sadlej, Chem. Phys. Lett. 112, 1 (1984).

[32] H.-G. Voelk and D. Fick, Nucl. Phys. A 530, 475 (1991).

[33] E. Arnold, J. Bonn, A. Klein, R. Neugart, M. Neuroth, E. W. Otten, P. Lievens, H. Reich, W. Widdra, and ISOLDE Collaboration, Phys. Lett. B 281, 16 (1992).

[34] A. Beckmann, K. D. Bökklen, and D. Elke, Z. Phys. 270, 173 (1974).

[35] R. Neugart, Z. Phys. 261, 237 (1973).

[36] E. Arnold, J. Bonn, R. Gegenwart, W. Neu, R. Neugart, E.-W. Otten, G. Ulm, K. Wendt, and ISOLDE Collaboration, Phys. Lett. B 281, 16 (1992).

[37] I. Tanihata, Phys. Lett. B 206, 592 (1988).

[38] K. Pachucki and J. Sapirstein, J. Phys. B (to be published); see also K. Pachucki, J. Phys. B 31, 5123 (1998). 